\title{
Antituberculosis drug resistance: practical solutions to practical problems
}

In tuberculosis (TB) programmes across the world, short-course chemotherapy using rifampicin has been shown to be one of the most effective and costeffective health interventions in the entire health sector [1]. Despite this, and the fact that we have had the drugs and knowledge to cure TB for years, the number of TB cases in the world is still likely to rise in the next decade [2]. We have known why drug resistance develops and how it can be prevented for decades, but in the last few years outbreaks of TB resistant to all the drugs in standard use have been reported by several countries. The outlook for patients with multi-drug resistant disease is poor, their chances of being cured are low, and as treatment is much more expensive [3], health services will not be able to treat them effectively. They will, nevertheless, consume considerable resources and will probably transmit infection with resistant strains to others before they die.

The number of people who develop active tuberculosis each year is likely to rise from 7.5 million in 1990 , to over 10 million by the year 2000 [2]. The principal reasons for this are the global population increase, poverty, inefficient TB programmes that do not stop transmissions of the disease, and the HIV epidemic. The greatest burden of disease $(>90 \%)$ is in developing countries, but this is a global problem. With increasing foreign travel, much of the disease now seen in developed countries has been acquired overseas. If the infection is acquired in an area where there are high levels of drug resistance, then there is a significant chance that the disease that subsequently develops will be resistant.

The introduction of streptomycin in the mid-1940s offered the first hope of really effective treatment, but when it was administered in isolation, streptomycinresistant organisms could be demonstrated in $80 \%$ of patients within 3 months [4]. This was because the bacterial load within a tuberculous cavity is such that among the $10^{8}$ sensitive organisms, $10-100$ streptomycin-resistant mutants will by chance be present. Treatment with streptomycin results in the selective proliferation of these organisms. Once $p$-aminosalicylic acid and isoniazid became available in 1946 and 1952 respectively, it became possible to suppress these mutants, as although $10^{-6}$ bacteria may be resistant to one drug, only $10^{-12}$ are likely to have spontaneous resistance to two and an even smaller fraction to three. For the first time it became possible to cure TB and avoid propagating organisms resistant to the drugs used [5-7].

The same principle underlies current strategy for TB treatment. Now, as then, when it is ignored, resistant organisms will be selected and then transmitted. While the bacterial load is high, in the first 2 months of treatment, new cases should receive at least three, and preferably four, drugs. The drugs used are rifampicin, isoniazid, pyrazinamide and ethambutol (or streptomycin). It is then necessary to continue treatment with two drugs to ensure that all bacteria are eliminated and the risk of relapse is minimised. If rifampicin and isoniazid are used, this continuation phase should last for 4 months. Continuation phase treatment with ethambutol and isoniazid should last for 6 months. The risk of failure due to primary resistance is extremely low with this regimen.

Patients who have received treatment before may exhibit acquired drug resistance. In most situations it is not feasible to perform susceptibility testing before re-treatment. The standard re-treatment regimen should be used, which will minimise the risk of failure due to acquired resistance to isoniazid and streptomycin. Five drugs - streptomycin, rifampicin, isoniazid, ethambutol and pyrazinamide - are administered under close supervision for the initial 3 months, followed by three drugs similarly supervised for a further 5 months. In well-run programmes the risk of failure or relapse following this regimen is $<10 \%$ [8]. A fundamental rule in the management of patients who may have drug resistance is that one should never add a single drug to a failing regimen [9].

Abuses of anti-TB drugs occur at many levels as described below.

Physicians may prescribe ineffective, non-standard anti-TB regimens, or may prescribe rifampicin for non-TB uses such as the treatment of sexually transmitted disease. One study in Bombay showed that 100 private practitioners were using 80 different regimens to treat TB [10]. In a study of American patients with multi-drug resistance, errors in previous management were detected in $80 \%$, with an average of 3.9 errors per patient [11]. 
Patients may take only some of the tablets prescribed (even when they are allegedly being 'supervised') and may fail to complete their course of treatment [12]. In many health services, pills are supplied loose, and dispensed into a screwed up piece of paper. Even in developed countries, patients often do not take the right number of pills in the right combination. As patients may be taking over 10 pills/day, it is not surprising that this is a major source of patients' complaints and confusion. Where there is a black market for TB drugs, patients may sell some of their medication, even if it is supplied free of charge, or may distribute some to other family members.

Pharmacists may inadvertently make errors in dispensing and calculating doses. Alternatively, they may supply the incorrect medication when certain drugs are out of stock, or when the patient cannot afford them.

Drug manufacturers may produce drugs with poor bioavailability, especially when three drugs are combined [13], as this may be associated with a marked decrease in the bio-equivalence of rifampicin. Poor drug quality with subtherapeutic dosages and counterfeit drugs are additional threats: if some drugs have no active ingredients then patients are effectively receiving mono or dual therapy.

Programmes may be poorly organised, with inadequate resources and logistics. This has resulted in the emergence of resistance in developing countries, like India, and in more developed areas such as New York. When antituberculosis chemotherapy has been inappropriately used for several years, levels of primary multidrug resistance may be as high as $7.5 \%$. By contrast, where there has been a consistently well-run programme multi-drug resistance in new cases should be $\leqslant 1 \%$ [14].

\section{Possible solutions}

Just as the problems occur at many levels, and are very often due to practical problems, rather than technical or scientific failings, a range of imaginative solutions that address the shortcomings of individuals and health systems will be necessary. New drugs for TB are unlikely in the near future, and would, in any case, offer only a temporary solution before further resistance develops unless effective strategies to prevent resistance are in use. In many countries all the following interventions will be necessary to prevent resistance levels from escalating.

Effective national programmes are necessary to prevent transmission within the community. Clear protocols, incorporating standardised drug regimens used by properly trained staff, maximise the chances that all patients will receive effective treatment. The national programme should supply adequate quantities of high quality drugs for these regimens to be used effectively. Use of sputum microscopy increases the specificity of TB diagnosis, and ensures that priority is given to the most infectious cases. Monitoring sputum conversion rates and the results of treatment demonstrates the effectiveness of treatment [15]. Improving the quality of the programme decreases the prevalence of resistant strains, as has been seen in Korea, Beijing and New York [16-18].

Direct observation of the patient as they take their medication improves adherence to the prescribed regimen and provides less opportunity for resistance to develop. Introduction of directly observed therapy in Tarrant County, Texas, resulted in the levels of drug resistance falling [19].

Packaging all drugs in combipacks makes it easier for patients to adhere to the treatment that has been prescribed, but there are still many pills to be taken and the possibility for selectively omitting certain drugs remains. If drugs are physically combined in the same preparation, then they cannot be used in isolation (by either patient or provider), and the subsequent emergence of resistant organisms is reduced. Physicians are more likely to prescribe an effective regimen, and the opportunity for inadvertent medication errors is decreased. Fixed dose combination drugs also simplify many of the logistical problems which contribute to drug shortages and the development of drug resistance in many countries. Despite all these benefits, use of triple dose combination drugs is limited in most of the world, although they are widely used in the UK. This is partly due to concerns about the bio-availability of rifampicin, partly because of cost concerns, and in some places because of concerns about 'clinical freedom'.

When drugs are physically combined in the same tablet, the bio-availability of rifampicin can decrease markedly. Although the bio-equivalence is acceptable in many preparations $[20,21]$, and these drugs achieve equivalent or superior results in clinical practice [22], it is likely that many of the currently available products are actually delivering subtherapeutic doses of rifampicin. A network of independent WHO coordinated quality control laboratories is therefore planned to ensure that the bio-equivalence of all combination chemotherapy in use can be monitored.

Currently, combination chemotherapy is more costly. However, prices may fall if manufacturers are ensured large orders, and combination drugs are perceived to be the treatment of choice. From a programme's point of view, the initial extra costs may be offset by much lower costs for the re-treatment of cases with resistant organisms. Given that adherence to standard regimens is one of the key factors in ensuring successful treatment, and 'adjustment by clinicians' is rarely indicated, the loss of flexibility associated with using fixed dose combination drugs is rarely a problem [23]. 
The introduction of a four-drug combination tablet would simplify management for both the individual and the programme. This would be a major development in the management of TB, and probably in the prevention of drug resistance.

In 1993, WHO declared TB to be a 'global emergency' because of the burden of disease and because of the threat of drug resistance. In most other areas industry has kept up with antimicrobial resistance by the production of new drugs, but there have been no new, effective antituberculous agents for the last 30 years, and it is unlikely that there will be any new potent and widely affordable drugs in the next 10-20 years.

We have a window of opportunity while we still have effective tools to treat patients, to decrease the burden of infection and disease. There is no single solution, but with good practice at all levels, and more widespread use of available strategy such as fixed dose combination drugs, it should be possible to cure TB without producing resistance. If we waste this opportunity with poor practices, delay and lack of commitment, multi-drug-resistant TB will become widespread and thousands of patients will become effectively untreatable. Given that national programmes in many developing countries are already going to be under pressure because of the increased number of patients if the only available and effective drugs lose their potency, these programmes will quite simply not be able to cope, and an emergency will become a disaster.

ELIZABETH TAYLER

Tuberculosis Programme, World Health Organization, 20 Avenue Appia,

CH-1211 Geneva 27, Switzerland.

\section{References}

1. World Bank. World development report: investing in health. New York, Oxford University Press. 1993: 116.

2. Dolin PJ, Raviglione MC, Kochi A. Global tuberculosis incidence and mortality during 1990-2000. Bull World Health Organ 1994; 72: 213-220.

3. Chaulet P, Raviglione M, Bustreo F. Epidemiology, control and treatment of multidrug-resistant tuberculosis. Drugs 1996; 52 (Suppl. 2): 103-108.

4. Medical Research Council. Streptomycin treatment of pulmon- ary tuberculosis. BMJ 1948; 2: 769-782.

5. Lehmann J. Para-aminosalicylic acid in the treatment of tuberculosis. Lancet 1946; 1: 15-16.

6. A Medical Research Council Investigation. Treatment of pulmonary tuberculosis with streptomycin and para-aminosalicylic acid. $B M J 1950 ; 2$ : 1073-1085.

7. A report to the Medical Research Council by their Tuberculosis Chemotherapy Trials Committee. Long-term chemotherapy in the treatment of chronic pulmonary tuberculosis with cavitation. Tubercle 1962; 43: 201-267.

8. Mazouni L, Zidouni N, Boulahbal F, Chaulet P. Treatment of failure and relapse cases of pulmonary tuberculosis within a national programme based upon short course chemotherapy. TRSU Progress Report 1992; 1: 36-42.

9. Crofton J, Chaulet P, Maher D. Guidelines on the management of drug resistant tuberculosis. WHO/TB/96.210. World Health Organization, Geneva. 1996.

10. Upplekar MW, Shepard DS. Treatment of tuberculosis by private general practitioners in India. Tubercle 1991; 72: 284-290.

11. Mahmoudi A, Iseman MD. Pitfalls in the care of patients with tuberculosis. Common errors and their association with the acquisition of drug resistance. JAMA 1993; 270: 65-68.

12. Sumartojo E. When tuberculosis treatment fails. A social behavioural account of patient adherence. Am Rev Respir Dis 1993; 147: 1311-1320.

13. Acocella G. Human bioavailability studies. Bull Int Union Tuberc Lung Dis 1989; 64: 38-40.

14. Cohn DL, Bustreo F, Raviglione MC. Drug-resistant tuberculosis: review of the worldwide situation and the WHO/IUATLD global surveillance project. Clin Infect Dis 1997; 24 (Suppl. 1): S121-S130.

15. Nuyangulu DS, Nkhoma WN, Salaniponi FML. Factors contributing to a successful control programme in Malawi. Bull Int Union Tuberc Lung Dis 1990; 66 (Suppl. 45-46): 45-47.

16. Kim SJ, Hong YP. Drug resistance in Mycobacterium tuberculosis in Korea. Tuber Lung Dis 1992; 73: 219-224.

17. Zhang LX, Kan GQ, Tu DH, Li JS, Liu XX. Trend of initial drug resistance of tubercle bacilli isolated from new patients with pulmonary tuberculosis and its correlation with the tuberculosis programme in Beijing. Tuber Lung Dis 1995; 76: $100-103$

18. Frieden TF, Fujiwara PI, Washko RM, Hamburg MA. Tuberculosis in New York City. Turning the tide. $N$ Engl $J$ Med 1995; 333: 229-233.

19. Weis SE, Slocum PC, Blais FX et al. The effect of directly observed therapy on the rates of drug resistance and relays in tuberculosis. $N$ Engl $J$ Med 1994; 330: 1179-1184.

20. Ellard GA, Ellard DR, Allen BW et al. The bioavailability of isoniazid, rifampin, and pyrazinamide in two commercially available combined formulations designed for use in the shortcourse treatment of tuberculosis. Am Rev Respir Dis 1986; 133: $1076-1080$.

21. Acocella G, Luisetti M, Grassi GG, Peona V, Pozzi E, Grassi C. Bioavailability of isoniazid, rifampicin and pyrazinamide (in free combination or fixed-triple formulation) in intermittent antituberculous chemotherapy. Monaldi Arch Chest Dis 1993; 48: 205-209.

22. Geiter LJ, O'Brien RJ, Combs DL, Snider DE. United States Public Health Service Tuberculosis Therapy Trial 21: preliminary results of an evaluation of a combination tablet of isoniazid, rifampin and pyrazinamide. Tubercle 1987; 68 Suppl 2: $41-46$.

23. Sbarbaro JA. A challenge-to our practices and to our principles. Tuber Lung Dis 1996; 77: 2-3. 